\title{
Joint Hypermobility in Schizophrenia: A Potential Marker for Co-Morbid Anxiety
}

\author{
Antonio Bulbena ${ }^{*}$, a , Lili Sperry ${ }^{\mathrm{a}}$, Bosco Anguiano ${ }^{\mathrm{b}}$, Guillem Pailhez and Jordi Gago ${ }^{\mathrm{c}}$ \\ ${ }^{a}$ Institut d'Atenció Psiquiátrica, Salut Mental i Toxicomanies, Psychiatry Department, Hospital del Mar, Universitat \\ Autonoma Barcelona, Spain \\ ${ }^{b}$ Ortuella Mental Health Center, Vizcaya, Spain \\ ${ }^{c}$ Sant Pere de Riudebitlles Primary Health Center, Barcelona, Spain
}

\begin{abstract}
Introduction: The objective is to evaluate the presence and clinical relevance of the Joint Hypermobility Syndrome (JHS) among patients with schizophrenia.

Methods: One hundred and twenty four outpatients with DSM-IV diagnosis of schizophrenia were assessed by means of the following: Hospital del Mar criteria for joint Hypermobility (JHdMar), Positive and Negative Syndrome Scale (PANSS), Fear Survey Schedule (FSS) and Social Adjustment Scale (SAS).

Results: JHS correlated positively with FSS $(r h o=0.36 \mathrm{p}<0.0001)$ and negatively with age $(\mathrm{rho}=-0.34 ; \mathrm{p}=0.0001)$. When taken as a dichotomic variable, PANSS-P (positive subscale) scores were significantly higher in the group with JHS as well $(Z=2.0 ; p=0.045)$. Subjects with co-morbid panic disorder scored significantly higher in the JHdMar. JHdMar scores were linked by stepwise multiple regression to panic disorder, positive symptoms, and age(-).

Conclusion: Joint hypermobility is significantly related to panic anxiety, positive symptoms and age in patients with schizophrenia. It is a probable clinical biological marker of interest.
\end{abstract}

Keywords: Joint hypermobility, schizophrenia, panic, anxiety.

\section{INTRODUCTION}

The joint hypermobility syndrome has been found to be associated with panic-phobic anxiety disorders in different kinds of samples such as rheumatologic outpatients, psychiatric outpatients and also non-clinical subjects $[1,2]$. Joint hypermobility is a benign heritable disorder of the connective tissue characterized by increased distensibility of joints It is more frequent in women $(3: 1)$ and has an estimated prevalence of $10-15 \%$ in the general population $[1,3]$. In schizophrenia, only isolated reports describe associated heritable collagen disorders such as temporomandibular joint dysfunction [4,5] and Marfan's syndrome [6,7]. Given that co-morbid anxiety has been found in schizophrenia with a prevalence between $30 \%[7,8]$ and $45 \%$ [8-10], according to different studies, and that the relationship between joint hypermobility and anxiety has been only preliminary reported in schizophrenic samples [7-11], new data on this association and its clinical correlates is offered in this study.

The Aim of the study is to report clinical correlates of the Joint Hypermobility Syndrome (JHS) in a sample of schizophrenic outpatients with the aim of providing information on a possible biological trait like an endophenotype that could be potential clinical marker.

\section{METHODS}

The study sample consisted of 140 patients with a diagnosis of schizophrenia (DSM-IV criteria) recruited conse-

*Address correspondence to this author at the Institut d'Atenció Psiquiátrica, Salut Mental i Toxicomanies, Psychiatry Department, Hospital del Mar, Universitat Autonoma, Barcelona, Spain; E-mail: abulbena@acmcb.es cutively from the outpatient clinic of a general hospital and a mental health centre in Bilbao, Spain. Subjects were aged between 18 and 60 years old and were receiving pharmacological treatment for their condition. Including only patients on antipsychotic medication was done to have a more homogenous group of patients with schizophrenia. Subjects were excluded from the study if found suffering from: 1 . an acute psychotic state, 2 . organic cerebral disease, 3 . concurrent severe substance abuse with the exception of nicotine, and 4. any musculoskeletal or rheumatologic conditions that hindered assessment. Sixteen subjects were excluded: 4 refused to participate, 4 were not taking antipsychotic medication; 2 exhibited an acute psychotic state; 4 were cocaine and heroine abusers; and 2 were suffering from joint inflammation in the knees and ankles. One hundred and twenty four subjects were included in the final sample. All participating subjects gave verbal consent and the study was approved by the University Ethics Research Committee.

Patients were interviewed by trained psychiatrists that administered the clinical and psychometric scales. Data was collected for the following socio-demographic variables: age, gender, marital status, years of education, lifetime cumulative hospitalization in months and age of disease onset. Data on medication used was also recorded.

Although diagnostic issues are dealt with in a separate paper, to assess the presence of any of the panic/phobic anxiety cluster disorders the structured clinical interview for DSM-IV (SCID) was used. In addition, clinical variables were evaluated. The Spanish version of the Positive and Negative Syndrome Scale (PANSS) was administered to determine if predominant positive or negative presentation of 
the illness relates to joint hypermobility. This scale is useful because it provides a bi-dimensional assessment and therefore permits correlations between the somatic variable and each of the dimensions. A modified 100-item version of the Wolpe Fear Survey Schedule (FSS) was administered to evaluate if fears are more frequent and/or intense in the group with JHS [12]. The Spanish version of the Social Adjustment Scale (SAS) was also used.

Following psychiatric evaluation patients were assessed independently for the physical variables by other trained clinicians blind to psychiatric diagnosis. Joint Hypermobility Syndrome was examined using Hospital del Mar (HdM Scale) criteria (Table 1; Fig. 1). This scale has been validated by this group [13] and is based on the more commonly known Beighton criteria but includes a few items more. Cutoff scores of $5 / 4$ for females and $4 / 3$ for males on the 10 point HdM Scale were considered diagnostic for joint hypermobility. Specific training for joint hypermobility assessment took place before the study began. Kappa values obtained by independent and trained ratters, for each joint item and for the total score, ranged between 0.65 and complete agreement for both JHS criteria.

Non-parametric Mann-Whitney and Spearman's rho were used for individual variable comparisons, since Gaussian distribution cannot be assumed for JHS. Correction for multiple comparisons was applied throughout. Finally to identify predictive independent variables, a stepwise multiple regression statistic was used. Data were kept on an Apple Macintosh G5 and JMP 5.1.1 (SAS Institute Inc.) software was used for the analysis.

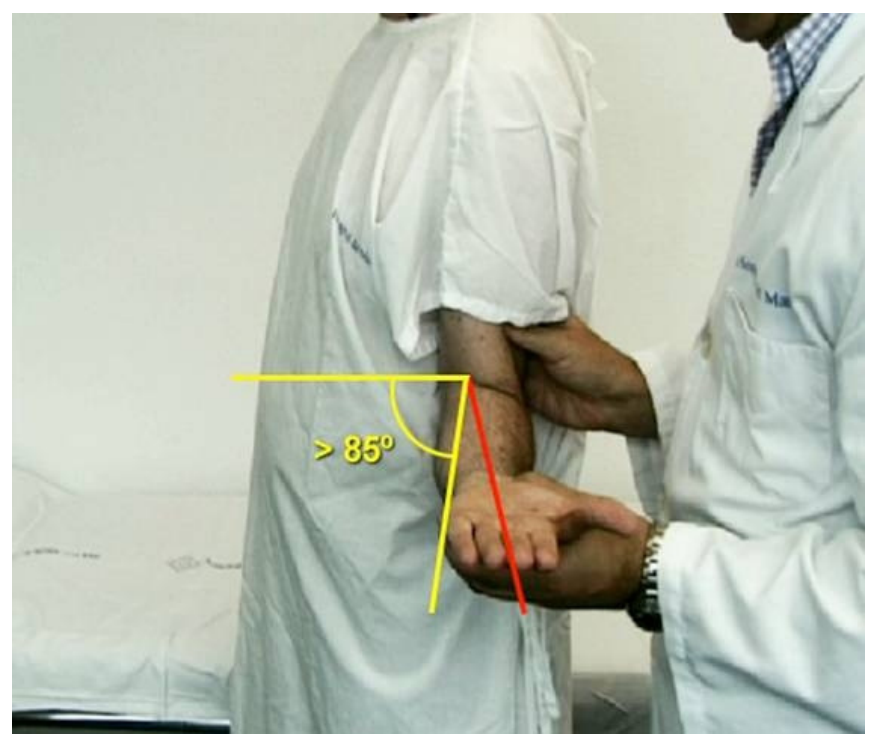

Fig. (1). Fifth H. Mar Criteria: External rotation of shoulder $>85^{\circ}$. Manouver: Bend the arm at a right angle and rotate forearm externally holding elbow at the side.

\section{RESULTS}

\section{Descriptive}

The sample included 67 males and 57 females with a mean age of $32.7( \pm 9.4)$ years for males and $34.6( \pm 10.8)$ years for females. Mean age of onset of disease was 24.5 $( \pm 6.8)$ years. $12.9 \%$ had never been hospitalized, and $58.8 \%$ had been hospitalized for no more than 3 months.
No significant differences were found between men and women for most variables. Males scored significantly higher for negative symptoms (PANSS-N) $(Z=-2.39 ; p=0.016)$ and females scored higher in the FSS $(Z=1.99 ; p<0.046)$.

Table 1. Hospital Del Mar Criteria for Joint Hypermobility Syndrome

\begin{tabular}{|l|l|}
\hline \multicolumn{2}{|c|}{ Joint Criterion } \\
\hline \hline Thumb & Apposition to forearm \\
\hline MCPhalangeal & Hyperextension of 5th finger past $90^{\circ}$ \\
\hline Elbow & Hyperextension $\geq 10^{\circ}$ \\
\hline Ankle/foot & Dorsiflexion $\geq 45^{\circ}$ in standing squat* \\
\hline Shoulder & External rotation $\geq 85^{\circ}$ from neutral \\
\hline Hip & Abduction $\geq 85^{\circ}$ \\
\hline Patella & Medial to lateral glide $\geq 1 / 2$ \\
\hline MTphalangeal & Dorsiflexion $\geq 90^{\circ}$ \\
\hline Knee & Flexion heel to contact buttocks \\
\hline Ecchymoses & Ecchymoses after minimal trauma \\
\hline
\end{tabular}

Even though it is not the focus of this article, it might be useful to underline that the prevalence of total anxiety disorders was $29.8 \%$ (37 patients) in this sample of patients with schizophrenia and that of these, $13.7 \%$ (17 patients) presented a panic/agoraphobia disorder [11].

\section{Direct Correlations}

Scores for Joint Hypermobility correlated positively with FSS (rho 0.36; $<<0.0001$ ) and negatively with age (rho $0.34 ; \mathrm{p}=0.001)$. However, no significant correlation was found for age onset, education, years of hospitalization and social adjustment. PANSS-P (positive subscale score) almost reached significance. Subjects with co-morbid panic/agoraphobia disorder scored significantly higher in the JdM (median 6) than those without co-morbid panic disorder (median 4) (Mann Whitney $\mathrm{p}<0.0001$ ).

When comparing groups with or without JHS by applying the cut-off scores and using it as a dichotomic variable, PANSS-P scores were significantly higher in the hypermobile group $(Z=2.0 ; p=0.045)$ and age significantly lower $(Z=-$ $2.03 ; p=0.042)$. FSS scores almost reached a significant difference $(Z=1.93 ; p=0.053)$.

\section{Stepwise Regression Models}

A logistic stepwise regression model was built for Joint Hypermobility (JHdMar). The JHdMar variable was included as a dichotomic dependent variable, the following variables were significant $\left(\mathrm{Chi}^{2}=25.89 ; \mathrm{p}<0.0001\right)$ : Comorbid Panic $(\mathrm{p}=0.0002)$, PANSS-P (positive subscale score $)(p=0.0269)$ and Age (negative) (0.0397).

\section{DISCUSION}

Our results show that the association between Joint Hypermobility and Anxiety in Schizophrenia is similar to the association found in non-clinical populations and in patients with anxiety disorders. In subjects with Schizophrenia, Joint 
Hypermobility is associated with younger age, panic anxiety, positive symptoms and higher scores in the fear survey.

The explanation for this JHS/anxiety relationship still remains unclear, albeit a genetic common ground has been published [14]. In a recent study carried out in a rheumatologic outpatient clinic, the majority of JHS patients presented various dysautonomic symptoms such as palpitations, chest discomfort, shortness of breath, light-headedness and flushing. These manifestations may overlap, or more likely, they coincide with those of the anxiety conditions. In another study, schizophrenic patients with high autonomic activity presented more positive symptoms and more severity. Unfortunately, anxiety conditions were not specifically addressed in that study [15]. Finally it has been reported that subjects with paranoid schizophrenia are more likely to suffer panic [16] and social anxiety even when controlling for hallucination and delusion related anxiety [17]. This was indirectly confirmed in our previous study [11], but interestingly JHS was also associated to higher PANSS-P scores. This seems to point in the direction of a cluster of panic symptoms, JHS and schizophrenia with predominant positive symptoms.

The limitations of our study must be commented. First, no control group was included; secondly, no biological, genetic or neuro-imaging variables were included in the comparison; thirdly, some clinical conditions were represented by scores in rating scales and hence they are more constructs than full clinical conditions.

The associations hypothesized in this paper have been broadly confirmed and indicate that the JHS could be a potential clinical marker among these patients. For example, a subject suffering from both Schizophrenia and Joint Hypermobility could have a higher chance of developing comorbid anxiety. Co-morbid anxiety in schizophrenia is known to correlate with severe positive manifestations, suicidal ideation, higher service utilization, and less quality of life. Therefore, this somatic trait can be of value in identifying subgroups of schizophrenia.

Mitral Valve Prolapse, a single manifestation of a benign connective tissue disorder and closely related to JHS was found associated to anxiety in the past. Perhaps JHS and MVP share an underlying collagen alteration and also a relationship to anxiety. This surprising link might be also found in schizophrenia, as our data suggest, but also partially found in a case report [18]. Perhaps studies following these lines of research can provide new findings that support their role as clinical biological makers.

\section{REFERENCES}

[1] Bulbena A, Duro JC, Porta M, et al. Anxiety disorders in the joint hypermobility syndrome. Psychiatry Res 1993; 46(1): 59-68.

[2] Martin-Santos R, Bulbena A, Porta M, Gago J, Molina L, Duro JC. Association between joint hypermobility syndrome and panic disorder. Am J Psychiatry 1998; 155(11): 1578-83.

[3] Bulbena A, Agullo A, Pailhez G, et al. Is joint hypermobility related to anxiety in a nonclinical population also? Psychosomatics 2004; 45(5): 432-7

[4] Kaban LB, Belfer ML. Temporomandibular joint dysfunction: an occasional manifestation of serious psychopathology. J Oral Surg 1981; 39(10):742-6.

[5] Perrini F, Tallents RH, Katzberg RW, Ribeiro RF, Kyrkanides S, Moss ME. Generalized joint laxity and temporomandibular disorders. J Orofac Pain 1997; 11(3): 215-21.

[6] Kalsi G, Mankoo BS, Brynjolfsson J, et al. The Marfan syndrome gene locus as a favoured locus for susceptibility to schizophrenia. Psychiatr Genet 1994; 4(4): 219-27.

[7] Sirota P, Frydman M, Sirota L. Schizophrenia and Marfan Syndrome. Br J Psychiatry 1990; 157: 433-6

[8] Argyle N. Panic attacks in chronic schizophrenia. Br J Psychiatry 1990; 157: 430-3.

[9] Cosoff SJ, Hafner RJ. The prevalence of comorbid anxiety in schizophrenia, schizoaffective disorder and bipolar disorder. Aust N Z J Psychiatry 1998; 32(1):67.

[10] Goodwin R, Davidson L. Panic attacks in psychosis. Acta Psychiatr Scand 2002; 105(1): 14-9.

[11] Bulbena A, Anguiano B, Gago, et al. Panic/Phobic Anxiety in Schizophrenia: A positive association with Joint Hypermobility Syndrome. Neurol Psychiatr Brain Res 2005 12: 95-100.

[12] Bulbena A, Gago J, Sperry L, Berge D. The relationship between frequency and intensity of fears and a collagen condition. Depress Anxiety. 2006; 23(7): 412-7.

[13] Bulbena A, Duro J, Porta M, Faus S, Vallescar R, Martin-Santos R. Clinical assessment of Hypermobility of Joints: Assembling criteria. J Rheumatol 1992; 19:115-122.

[14] Gratacós M, Nadal N, Martín-Santos R, et al. A polymorphic genomic duplication on human chromosome 15 is a major susceptibility genetic factor for panic and phobic disorder. Cell 2001; 106: 367-379.

[15] Zahn T, Picar D. Autonomic activity in relation to symptom ratings and reaction time in unmedicated patients with schizophrenia. Schizophr Res 2005; 257-270.

[16] Labbate LA, Young PC, Arana GA. Panic disorder in schizophrenia. Can J Psychiatry 1999; 44(5):488-90.

[17] Tibbo P, Swainson J, Chue P, LeMelledo JM. Prevalence and relationship to delusions and hallucinations of anxiety disorders in schizophrenia. Depress Anxiety 2003; 17(2): 65-72.

[18] Sienaert P, De Hert M, Houben M, et al. Safe ECT in a patient with the Ehlers-Danlos syndrome. J Ect 2003; 19(4): 230-3. 